\title{
DES APPAREILS DE PASTEURISATION A PLAQUES
}

\author{
par Henri STASSANO.
}

Il y a vingt ans, au début de mes études concernant la détermination des températures les plus basses auxquelles on peut, par la chaleur, tuer les différentes espèces de microbes et la recherche d'un procédé thermique approprié pour ces déterminations et permettant d'atteindre les différentes espèces de microbes d'une façon sûre; uniforme, à leurs températures minima mortelles respectives, je faisais usage de dispositifs à plaques. Et il ne pouvait en être autrement, car je venais d'essayer la stérilisation des émulsions ou suspensions microbiennes capables de donner des vaccins plus actifs que les ordinaires, chauffées au bain-marie, ou des tests pour les réactions d'agglutination, presque aussi sensibles que ceux constitués par les microbes vivants, et de tenter également la stérilisation du lait, par les rayons ultraviolets, précisément en étalant sous une couche ultra-mince, derrière une lame de quartz rigoureusement aplanie, soit les suspensions microbiennes, soit le lait.

La lame de quartz était appliquée sur une autre lame en métal, aussi rigoureusement plane, de mêmes dimensions et de forme rectangulaire, lui étant en tout point superposable. Seul un cadre en papier japon, de 1/100 de millimètre d'épaisseur, était interposé entre les deux, en contournant le périmètre extérieur et limitant ainsi, en dedans, une sorte de cuve excessivement mince. A travers cette sorte de cuve, d'une épaisseur aussi mince que régulière, on faisait circuler les émulsions microbiennes ou le lait naturellement chargé de microbes, sous la poussée uniforme et réglable d'un gaz inerte, l'azote.

A cet effet, sur chacun des deux côtés les plus éloignés de cette cuve, au ras même du cadre, et naturellement en dedans, il y avait une rangée de petits trous en communication au-dessous avec un caniveau creusé dans l'épaisseur du métal ; à chaque rangée de trous correspondait un caniveau. La circulation était done établie d'un caniveau à l'autre, à travers pareille cuve, dont l'étanchéité parfaite était assurée en enduisant de vaseline le cadre en papier japon, légèrement gaufré, et en serrant par-dessus, tout autour des bords, la lame de quartz contre la plaque métallique à l'aide de boulons à oreilles, disposés à intervalles égaux et très rapprochés.

En remplaçant peu après l'action des rayons ultraviolets par celle des radiations thermiques, le dispositif resta le même, sauf que la lame de quartz fut remplacée par une lame métallique, en bronze. Celle-ci était parfaitement superposable à la plaque portant le cadre en papier japon. Réunies, elles formaient un bloc unique que l'on plongeait dans un bain-marie, lors des essais. Ce bloc, avec les canalisations d'arrivée et de sortie des différents liquides sur lesquels 
portaient les recherches, était, bien entendu, stérilisé au préalable, pour éliminer les microbes qui le souillaient et n'avoir à faire qu'à une seule espèce microbienne : celle à l'étude.

Dans la suite, pour allonger le parcours, sans trop augmenter la longueur de chaque lame ou plaque métallique, j'adoptai le dispositif (fig. I) où l'on voit trois plaques superposées.

Le liquide passe de l'une à l'autre, un des caniveaux de la plaque du milieu mettant en communication directe les deux rangées de petits trous ouverts sur les deux surfaces opposées de la même plaque. Le chauffage de cette

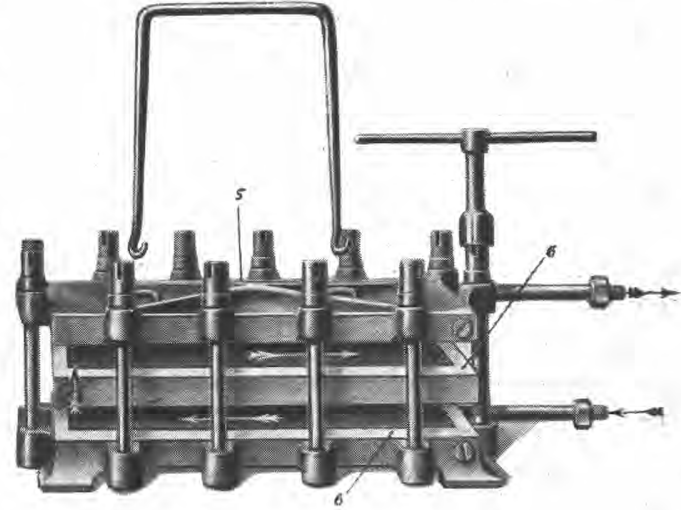

Fig, I, plaque moyenne se fait par ses côtés, assez larges, en contact avec l'eau du bain-marie.

Dans la figure II, sur l'appareil suspendu à un palan et prêt à être. plongé dans un autoclave particulièrement aménagé, on peut distinguer au niveau de la plaque du milieu (cet appareil étant également constitué de trois plaques superposées) une sorte d'ailette. Cette plaque du milieu en effet se termine en dehors de ses deux surfaces. absolument planes, rectangulaires, par une crête qui fait saillie tout autour de son périmètre, de façon à augmenter la surface de contact qu'elle peut avoir par les côtés avec l'eau du bain-marie. Je n'avais pas cru nécessaire de faire figurer ce troisième appareil dans mon mémoire paru en 1924 dans les Annales de l'Institut Pasteur, à la suite de la précédente figure, mais j'ai tenu, à présent, à en donner dans cet article la vue d'ensemble, - avec les différents organes auxquels il est relié : d'une part, l'autoclave pouvant fonctionner comme bain-marie, et, de l'autre, le réfrigérant avec le dispositif complet de prise d'échantillons et de distribution aseptique des liquides traités, selon une technique longuement étudiée, - pour cette raison particulière que voici : Par son mode de fixation au couvercle: de l'autoclave, lequel ici possède la forme d'une cuvette, l'appareil fig. II rappelle le tout premier dispositif à plaque que j'imaginai et fis fonctionner, en union avec un ingénieur de ma nationalité, M. Gовві (inventeur d'un très ingénieux filtre formé par une spirale en acier s'enroulant sur elle-même), à Asnières, dans un atelier 
d'essais, fondé par le propre fils du grand historien TAINe. Il l'avait appelé, avec humour, sinon avec prétention, "le berceau des inventione "

Notre très simple dispositif était constitué par deux plaques en

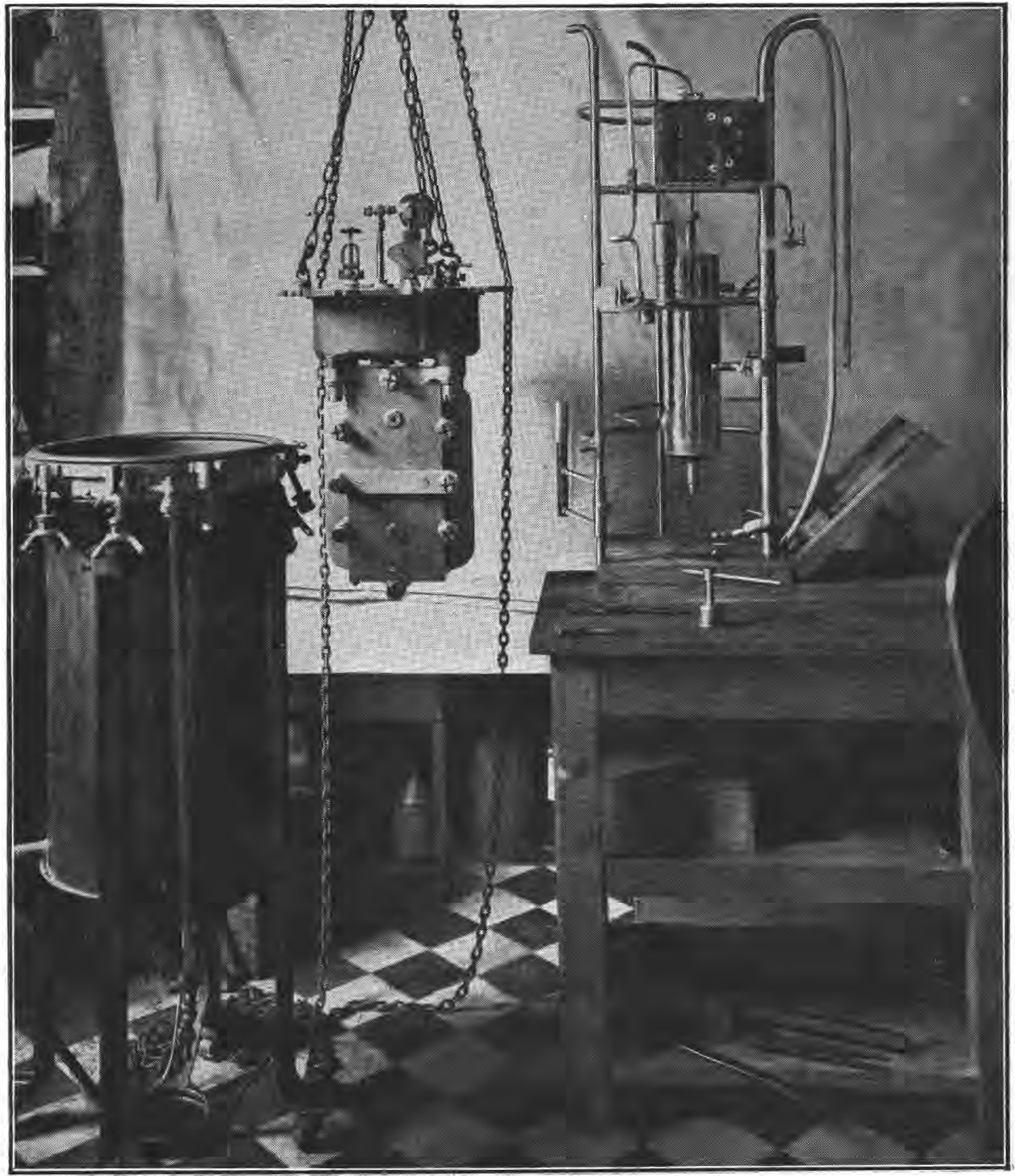

Fig. II.

fer-blanc, soudées l'une à l'autre tout autour avec un léger écartement entre elles. C'était en somme une boîte très aplatie, en communication, à l'un des bouts avec un tuyau, et à l'autre extrémité, sur le plan longitudinal, du même côté, avec une seconde tubulure. Dans cette 
boîte, on faisait couler du lait sous pression, et la longueur du parcours dans son intérieur, entre l'entrée et la sortie, était quadruplée, quintuplée, par un système de chicanes soudées intérieurement sur les parois des deux plaques. Les deux canalisations d'entrée et de sortie traversaient de part en part, comme dans l'appareil fig. II, le couvercle usuel d'un autoclave ordinaire.

La ressemblance entre le premier dispositif à plaques et cet appareil (fig. II) perfectionné que je pus construire beaucoup plus tard avec les larges moyens mis à ma disposition par l'Institut Pasteur, et que beaucoup de monde a vu à Paris dans mon laboratoire de la rue Dutot, à partir de 1915, est done très grande.

D’ailleurs, dans le brevet que je déposai le 23 mars 1911 en Belgique, je parle nettement : "d'un moyen propre à obliger ledit liquide à venir se soumettre à ladite source (rayons ultraviolets, chaleur, vapeur surchauffée, mélanges salins, bains d'huile ou de substances équivalentes, courant électrique, etc.) sous une épaisseur extrêmement réduite (toutes choses égales d'ailleurs), de façon qu'il ne soit besoin de soumettre le liquide à stériliser à l'action de la chaleur que pendant un temps relativement très faible" - et, ensuite, entrant dans les exemples des dispositifs qui peuvent permettre de réaliser l'invention dont il s'agissait, mon brevet envisage clairement le cas d' " un récipient plat, qu'on agence à l'intérieur d'un autoclave et auquel on fait avantageusement comporter, à la façon bien connue, des sortes de chicanes propres à assurer une circulation plus régulière du liquide à stériliser à son intérieur».

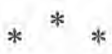

Dans mon mémoire plus haut cité des Annales de l'Institut Pasteur, j'ai expliqué pourquoi j'ai abandonné ces dispositifs à plaques et comment j'ai été amené, peu à peu, à réaliser la couche mince dans des conditions mieux appropriées à la transmission la plus rapide de la chaleur aux microbes, à la production la plos forte de l'effet de la paroi chauffante sur les microbes, qui viennent à son contact.

Ces conditions indispensables pour une bonne pasteurisation, je Ies ai réalisées en enchâssant un tube rigoureusement cylindrique dans un autre aussi rigoureusement cylindrique, concentriquement l'un à l'autre. Dans ce cas, si leurs diamètres diffèrent très peu l'un de l'autre, et si leur épaisseur est très réduite - un millimètre comme ils sont tous les deux également en cuivre, le métal usuel doué de la plus faible capacité calorifique, on est sûr d'atteindre les deux buts principaux, on peut même ajouter : les deux buts idéaux de la pasteurisation, malgré qu'ils soient presque opposés, antinomiques l'un à l'autre : l'effet maximum de la paroi chauffante, c'est-à-dire 
l'effet le plus meurtrier sur les microbes, avec le minimum de modification dans les constituants, parfois très délicats et fragiles au chauffage, du liquide où les premiers se trouvent, du liquide qui les véhicule et dont on doit l'en débarrasser par le traitement thermique en question.

$\mathrm{Au}$ contraire, dans tous les autres procédés, usuels et même les plus récents, de pasteurisation, la chaleur est utilisée comme dans une simple opération de cuisine : on sait que plus les aliments sont cuits, mieux ils se conservent et on pousse le chauffage plus haut ou on le maintient plus longtemps, ne s'arrêtant, dans l'une ou l'autre de ces poussées, que là où les modifications apportées par le chauffage commencent à devenir par trop évidentes ou nuisibles aux produits ainsi traités.

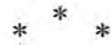

Le professeur S. ORLA-Jensen, dans un de ses derniers mémoires, cite mon procédé, en même temps que celui de la Biorisation, en leur appliquant l'épithète élogieuse de procédés «ingénieux ». Je ne pense pas que ce soit là le qualificatif qui convienne. Il y a plus d'ingéniosité dans nombre de jouets exposés chaque année au Concours Lépine, à Paris, que dans mon procédé. Il ne faut voir, dans celui-ci, autre chose que le long labeur d'un homme de science, habitué déjà depuis longtemps à la recherche dans plusieurs domaines de la science, ce qui est plutôt rare dans ce siècle de spécialisation à outrance. Quant au procédé de la biorisation, même en admettant qu'il donne des résultats bactériologiques aussi bons que le mien, comment lui appliquer cette épithète d'ingénieux, si en "biorisant", le lait, on lui fait perdre tout son acide carbonique et une partie appréciable de son volume, tout en mettant le lait dans les conditions les plus défavorables pour la conservation des vitamines : action du chauffage combinée à une très forte oxydation? Je ne parle pas de la mauvaise conservation des autres principes du lait, non moins précieux que fragiles, qui doit certainement accompagner la biorisation.

Au demeurant, s'il y a toutefois quelque ingéniosité dans ce procédé, je me refuse à y reconnaître la moindre originalité. Déjà, vers 1910, un constructeur parisien très renommé (spécialement pour ses prismes) d'appareils d'optique, WerLeIN, a pris un brevet pour un mode voisin de traitement du lait. Le lait devait se chauffer rapidement en traversant, sous pression, une sorte d'éponge métallique en bronze. Dès cette époque, je déconseillai WERLEIN de pousser plus loin ses efforts dans cette voie. Ensuite, on a construit des appareils sur le même principe de la pulvérisation d'un liquide, notamment pour sécher les sérums. 
Quoi qu'il en soit, pour exprimer une opinion sur la valeur de tel ou tel procédé industriel de pasteurisation, il faut tenir compte de cette, particularité essentielle d'une bonne pasteurisation : Ia conservation de l'acide carbonique dont le lait est encore chargé à son arrivée dans les laiteries.

J'ai appris à connaître l'importance de cette conservation il y a déjà plus de vingt ans dans les remarquables travaux d'un chimiste français très distingué, M. BARILLé. Sa première note : "Del'existence des carbono-phosphates dans le lait ; leur précipitation par la pasteurisation" (C. R. de l'Académie des Sciences, avril 1909) retint de suite mon attention. En ce moment, je m'occupais déjà de stériliser le lait, mais par les rayons ultraviolets. C'est cependant beaucoup plus tard que j'ai été amené, par mes propres recherches, à connaître et à apprécier les différents effets utiles de la conservation de l'acide earbonique. Elle ne se borne pas à diminuer, dans une très forte mesure, la formation de ce gênant dépôt qu'on appelle "la pierre de lait ", à base de phosphate tricalcique, sur les appareils usuels de pasteurisation ; elle fait beaucoup plus : elle conserve au Jait toute sa valeur alimentaire, en y maintenant à l'état soluble, et, par conséquent, assimilable, les sels de chaux et les phosphates et elle lui conserve simultanément toute l'efficacité que le lait possède à l'état cru comme vecteur de principes immunisants pour le tube digestif vis-à-vis des infections intestinales si fréquentes et meurtrières chez les enfants en bas âge. En effet, avec la conservation intégrale de l'acide carbonique agissant également sur les globulines, tous les délicats équilibres chimiques qui sont sous sa dépendance se maintiennent gardant leurs propriétés.

La démonstration de ces avantages, je l'ai donnée dans différentes publications, notamment dans la note communiquée à l'Académie des Sciences, en collaboration avec A.-P. RoLLET, de l'Institut de Chimie de l'Université de Strasbourg (C. R., tome CLXXIX, p. 297) : " De l'appauvrissement du lait en acide carbonique par la pasteurisation usuelle à l'air libre. Avantage du traitement en circuit fermé. "Et c'est depuis cette note et par elle que l'on a pu commencer à parler des avantages du circuit fermé dans la pasteurisation du lait.

Cependant, on y aurait pu songer bien avant, en ne considérant même que l'un d'entre eux: l'efficacité de l'action du chauffage sur les microbes. En effet, Russhil et Hastings ont fait connaître, déjà en 1902, qu'en vase clos (l'équivalent du circuit parfaitement fermé), on tue par la chaleur les microbes à 5 ou 6 degrés de température plus bas qu'à l'air libre (Centralblatt für Bakt. Paras- und Infekt., t. VIII, avril 1902). Mais ce fait de toute première importance passa inaperçu et, encore aujourd'hui, il est ignoré par les constructeurs d'appareils, même par ceux qui 
se sont mis, à ma suite, à construire des pasteurisateurs qu'ils croient comme les miens " en circuit fermé ", mais où ils intercalent inconsidérément des " holdings »!

Les deux notes que j'ai communiquées le 10 juillet 1925 à la Société de Biologie à la suite de la note ci-dessus citée de l'Académie des Sciences : $1^{\circ}$ Aération du lait pasteurisé ; $2^{\circ}$ Variations des équilibres chimiques du lait provoquées par le vide et le chauffage (pasteurisation), permettent de connaître et d'apprécier encore mieux les différents avantages d'ordre chimique du circuit fermé dans le traitement thermique du lait.

Les distingués professeurs de l'Institut de Laiterie de Kiel, dans l'étude attentive et minutieuse qu'ils ont faite, l'année dernière, comparativement sur les nombreux et différents appareils de pasteurisation dont disposait le très important Physikalisches Institut für Milchwissenschaft, n'ont nullement tenu compte de ce facteur essentiel d'une bonne et parfaite pasteurisation : la conservation de l'acide carbonique, signalée par les publications de BARILLé et les miennes. Je ne connais pas leur rapport officiel, mais, d'après le résumé que le professeur Dr HENNEBERG en a donné dans la conférence qui a été reproduite dans l'article Zweite Milchwirtschaftliche Woche in Kiel, par la revue Die Milchindustrie d'avril 1930, ce facteur n'entre pour rien dans le mode de classement par eux adopté.

Pour ce qui me concerne, je n'ai pas trop à me plaindre, car mon appareil occupe dans leur classement une place assez honorable, la troisième, malgré qu'ils aient complètement méconnu en lui cette prérogative essentielle du procédé de la couche vraiment mince, en circuit parfaitement fermé, d'assurer la conservation intégrale de l'acide carbonique dans le lait, conservation qui fait plus ou moins défaut avec les autres modes de traitement du lait par la chaleur. Je ferai remarquer en outre - ce qui a été vraiment regrettable - que l'appareil de mon système sur lequel a porté l'étude qui a été faite à Kiel (l'un des deux premiers appareils de ce système construit au Danemark, le deuxième ayant été l'appareil qui a servi aux essais de contrôle pratiqué pendant deux ans à la Station officielle danoise de Hilleröd) était en grande partie dépourvu de la qualité qui est précisément l'une des principales de mon procédé de pasteurisation, de donner un lait d'une très bonne conservation, parfaitement assaini et jouissant en plus de l'avantage de voir sa crème se séparer au repos, même un peu plus hâtivement que dans le lait cru témoin et en égale abondance.

Dans les appareils construits après et qui se trouvent déjà en fonction dans plus de vingt laiteries, ce défaut a été peu à peu amendé (1). Il tenait à ce que, dans l'appareil de Kiel, comme dans l'appa-

(1) Les deux premiers appareils de Hilleröd et de Kiel présentaient eneore beaucoup de difficultés pour le nettoyage. Peu à peu ces difficultés à lour tour ont été vaincues par les 
reil de Hilleröd, la récupération des calories était poussée beaucoup trop loin dans les éléments du récupérateur au détriment des qualités du lait. Et l'on comprendra facilement d'où venait cette erreur. Les plans de ces deux premiers appareils furent calqués, pour ainsi dire, sur le premier appareil construit, pour une laiterie d'Alger, par une fabrique d'appareils frigorifiques de Strasbourg. Les ingénieurs de cette usine, habitués aux ealculs de leurs appareils frigorifiques, malgré mes instructions particulières, exagérèrent précisément en retenant le lait trop longtemps chaud pour lui faire davantage céder sa chaleur.

Voici la classification des différents appareils de pasteurisation d'après le système de ponctuation adopté par l'Institut de. Kiel : Pasteurisation basse (type Astra, je erois) : 19 points, Electropasteurisation: 18, Biorisation : 15, Stassano: 15, A. P. V.: 13, Montana : 13, Todt: 12, der Ringscheibenpasteur: 7 , et Stabrührwerkpasteur: 5 . Cette dernière notation a été décernée à un appareil que j'ai vu fonctionner en Italie. Il s'agit d'une grande cuve dans laquelle le lait est chauffé par l'agitation d'un serpentin d'eau chaude qui tourne sans cesse et très rapidement, formant une épaisse couche de mousse, dont se dégage une buée très chaude. L'impression est d'avoir devant soi une chaudière de lait en ébullition, et pourtant le thermomètre n'accuse que $63^{\circ}$.

Je n'ai donc pas trop à me plaindre comme j'ai dit plus haut de la place qu'actuellement mon procédé occupe dans cette elassification. Toutefois, je me permets de faire constater ici qu'aucun des appareils, même parmi les mieux classés par l'Institut de Kiel, n'a subi le rigoureux contrôle dont le mien a été l'objet à Hilleröd, avec les quatre séries d'expériences exécutées par le Prof. Bang, de l'Ecole Supérieure Vétérinaire de Copenhague, sur du lait naturellement infecté de bacilles tuberculeux. Ne sait-on pas que les auteurs les plus récents déclarent notamment : $1^{\circ}$ que la pasteurisation haute ne donne aucune certitude d'assainissement du lait naturellement tuberculeux ; $2^{\circ}$ que cet assainissement peut être obtenu par la pasteurisation basse, avec cette restriction pourtant, à savoir : la pasteurisation basse, même avec les appareils les plus modernes, ne réussit pas à détruire dans tous les cas les bacilles de la tuberculose présents dans le lait. Telle est l'opinion, non seulement de Zeller, Wedemann, Lange, Gildemeister de la Mitglieder des Reichsgesundheitsamts - Berlin 1928, mais aussi du Dr Seelemann, de Kiel, Eickmann et Soentjen de Bonn, de Meantell - The Veterinary Record, 7 avril 1928 ; de L.C. Тноми́ - Swenska Mejeritidningen, 8 décembre 1928.

ateliers de Silkeborg. Je peux donc affirmer aujourd'hui qu'au plus faible dépôt que mon procédé produit sur les surfaces chauffantes correspond également une beaucoup plus grande facilité de nsttoyage, démontage et ràmontage. 


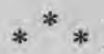

Après cette digression, - que j'ai senti le besoin de faire avant que paraisse mon prochain mémoire où « je dresserai la liste des différents et nombreux critériums qui seuls permettent, en en tenant compte dans la mesure que la signification et la portée que chacun exige, d'émettre un avis valable, solidement établi sur la valeur d'un nouveau procédé de pasteurisation en lui-même, ainsi que par comparaison avec les procédés existants ", - revenons à la partie essentielle de cet article : les appareils à plaques comme mode de réalisation de la pasteurisation en circulation continue sous couche mince.

L'efficacité particulière, vraiment élective sur les microbes du procédé thermique de la couche mince, tient essentiellement en ceci que, dans chaque unité de temps, un tout petit volume du liquide en voie d'être uniformément soumis à l'action de la chaleur se trouve réparti sur une très grande surface chauffante, constituée, dans l'espèce, par deux surfaces métalliques placées l'une en face de l'autre à la distance minime, et rigoureusement égale d'un bout à l'autre de $1 \mathrm{~mm}$. Ces deux surfaces, ainsi rapprochées entre elles, sont maintenues exactement, pendant les opérations, sur toute leur étendue, à la même température, laquelle peut être appropriée, soit à la pasteurisation, soit à la stérilisation.

C'est entre ce minime autant qu'uniforme interstice, séparant les deux surfaces chauffantes en question, que circule le liquide pendant le traitement, avec une vitesse constante. Celle-ci ne peut être ni inférieure à 1 mètre par seconde, sans nuire à la qualité, aux propriétés du liquide quel qu'il soit qui traverse l'appareil, et, d'autre part, ni supérieure à 2 mètres par seconde, sans risquer le bon succès de l'opération par le fait de laisser échapper vivants une partie des microbes qu'il faut atteindre.

De plus, et cela d'après mes propres déterminations aussi bien de laboratoire qu'industrielles, le parcours que les liquides doivent suivre entre les dites surfaces chauffantes sous l'épaisseur de 1 millimètre, ne peut pas être inférieur à la longueur de 10 mètres. Il y a même intérêt à allonger ce parcours afin d'être plus sûr encore de l'effet cherché.

Dans un appareil de mon système en fonction depuis trois ans en Algérie, l'écart entre les deux surfaces chauffantes a été porté de $1 \mathrm{~mm}$. à $3 \mathrm{~mm}$; - - la longueur du parcours du lait a été également accrue, mais seulement doublée. Malgré ces changements que le propriétaire de la laiterie a cru utile d'apporter pour faciliter le travail de la pompe - il ne s'est produit aucun échec en ce qui concerne la conservation du lait jusqu'au moment de la livraison et de la consommation chez les particuliers, bien qu'en Algérie on ait souvent des laits très acides, en voie de fermentation lactique à leur arrivée 
à la laiterie. Cependant, l'assainissement du lait a dû être maintes fois compromis, car on retrouvait encore dans le lait après le traitement, dans les conditions ci-dessus, des coli-bacilles vivants.

$\mathrm{Au}$ surplus, il ne suffit pas que la couche mince ne dépasse pas le millimètre et que le parcours du lait entre les surfaces chauffantes ne soit pas inférieur à 10 mètres, il faut encore : $1^{\circ}$ que l'épaisseur des parois métalliques soit de $1 \mathrm{~mm}$., pas davantage. Sur un appareil de mille litres de débit par heure, en changeant les premiers tubes que le constructeur avait employés et qui mesuraient $1 \frac{1}{2} \mathrm{~mm}$ d'épaisseur, j'ai pu constater immédiatement, par l'examen bactériologique direct du lait, que l'efficacité du chauffage s'était accrue sensiblement, toutes les autres conditions de l'opération n'ayant pas changé ; $2^{\circ}$ il est également nécessaire que le métal choisi pour ces surfaces ehauffantes, soit le cuivre rouge, e'est-à-dire le seul métal que l'on puisse facilement se procurer possédant la plus faible capacité calorifique, propriété essentielle pour assurer une transmission de la chaleur extrêmement rapide dans le contact infiniment bref qu'un microbe peut avoir, des millions de fois, avec les parois chauffantes. Les microbes, animés constamment du mouvement brownien, sont amenés contre ces parois (dans des contacts de l'ordre de grandeur et de durée des déplacements des bactéries) (1) par la force d'adhésion capillaire que ces mêmes parois exercent sur eux, le long du parcours qu'ils accomplissent contre elles, parois dont ils ne sont jamais plus éloignés de $5 / 10$ de $\mathrm{mm}$.

(A suivre.)

\title{
DU CHOIX D'UN ANIMAL RÉACTIF POUR L'ÉTUDE DE L'ÉQUILIBRE. NUTRITIF DES LAITS (a)
}

\author{
par
}

\section{Mme L. RANDOIN et M. R. LECOQ}

Utilisée seule, l'analyse chimique d'un aliment donné ne permet pas de connaître la valeur nutritive véritable de cet aliment ; il est maintenant prouvé que l'analyse chimique doit être complétée, dans tous les cas, par une analyse biologique faite avec plusieurs

(1) Par l'expression " ordre de durée des bactéries ", je ne me rapporte pas à la durée de la vie des bactéries; mais simplement : $a$ ) à la durée de leurs déplacements; $b$ ) à la durée néces saire pour produire chez elles telle ou telle autre manifestation, notamment la coagulation de leur albumine par l'effet du chauffage ; c) à la durée des contacts qu'elles peuvent avoir entre elles ou avec des objets extérieurs, contacts minimes, mais toujours appréciables, non de l'ordre des infiniment petits ainsi que la cinétique en prévoit entre corps infiniment petits non déformables.

(a) A paru dans les Annales de Falsifications et des Fraudes, 1930, p. 132. 